\title{
Inhibition of melanocortin-4 receptor dimerization by substitutions in intracellular loop 2
}

\author{
Carolin L Piechowski, Anne Rediger, Christina Lagemann, Jessica Mühlhaus, \\ Anne Müller, Juliane Pratzka, Patrick Tarnow, Annette Grüters, Heiko Krude, \\ Gunnar Kleinau and Heike Biebermann
}

Institute of Experimental Pediatric Endocrinology, Charité-Universitätsmedizin Berlin, Humboldt-Universität zu Berlin, Augustenburger Platz 1, 13353 Berlin, Germany

\begin{abstract}
Obesity is one of the most challenging global health problems. One key player in energy homeostasis is the melanocortin-4 receptor (MC4R), which is a family A G-protein-coupled receptor (GPCR). It has recently been shown that MC4R has the capacity to form homo- or heterodimers. Dimerization of GPCRs is of great importance for signaling regulation, with major pharmacological implications. Unfortunately, not enough is yet known about the detailed structural properties of MC4R dimers or the functional consequences of receptor dimerization. Our goal, therefore, was to explore specific properties related to MC4R dimerization. First, we aimed to induce the dissociation of dimers to monomers and to compare the functional parameters of wild-type and MC4R variants. To inhibit homodimerization, we designed MC4R chimeras with the cannabinoid-1 receptor, a receptor that does not interact with MC4R. Indeed, we identified several substitutions in the intracellular loop 2 (ICL2) and adjacent regions of transmembrane helix 3 (TMH3) and TMH4 that lead to partial dimer dissociation. Interestingly, the capacity for signaling activity was generally increased in these MC4R variants, although receptor expression remained unchanged. This increase in activity for dissociated receptors might indicate a link between receptor dimerization and signaling capacity. Moreover, dimer dissociation was also observed in a naturally occurring activating MC4R mutation in ICL2. Taken together, this study provides new information on the structural prerequisites for MC4R dimerization and identifies an approach to induce the dissociation of MC4R dimers. This might be useful for further investigation of pharmacological properties.
\end{abstract}

Correspondence should be addressed to H Biebermann Email heike.biebermann@charite.de

\author{
Journal of Molecular \\ Endocrinology \\ (2013) 51, 109-118
}

\section{Introduction}

The melanocortin-4 receptor (MC4R) is encoded by a single-exon gene and is primarily expressed in the hypothalamic paraventricular nucleus, spinal cord, sympathetic preganglionic neurons, brain stem, and penis (Van der Ploeg et al. 2002, Tao 2010). Upon activation by the pro-opiomelanocortin-derived peptides $\alpha$-MSH and
$\beta$-MSH, the receptor mainly couples to the Gs/adenylyl cyclase system, but also activates a variety of downstream effectors, such as kinases, leading to decreased food intake and increased energy expenditure (Cone 2006). From in vitro studies, it is known that MC4R exhibits ligandindependent activity (Nijenhuis et al. 2001). Heterozygous 
MC4R mutation is the most frequent known genetic cause of obesity in humans (Farooqi et al. 2003). Because of its important role in hypothalamic energy metabolism, the MC4R seemed to be a suitable target for pharmacological treatment, although effective targeting in humans has not yet been achieved (reviewed in Biebermann et al. (2012)). Currently known MC4R ligands cause decreased food intake in mice but not in humans (Krishna et al. 2009).

It has recently been shown that MC4R is able to form homodimers (Biebermann et al. 2003, Elsner et al. 2006, Nickolls \& Maki 2006) and that a few mutations cause dominant-negative effects on wild-type (WT) receptor function (Biebermann et al. 2003, Tarnow et al. 2008). Furthermore, heterodimerization has been demonstrated between MC4R and other G-protein-coupled receptors (GPCRs), such as GPR7, that are potentially involved in weight regulation (Rediger et al. 2009). Heterodimerization of MC4R has not yet been successfully considered in drug design (Rozenfeld \& Devi 2010, 2011).

Little is yet known about the structural and biophysical prerequisites for MC4R dimerization (reviewed in Rediger et al. (2012)). The impact (if any) on functional properties in comparison to MC4R monomers is still unclear, although it is well established that dimerization of many GPCRs can have a dramatic influence on the signaling properties of the interacting protomers - such as ligand binding (Levoye et al. 2006), G-protein coupling selectivity, or signal transduction mechanisms (reviewed in George et al. (2002)).

The current study was performed to obtain deeper insights into the prerequisites and consequences of MC4R homodimerization. For simplicity, we will use the term dimerization, although we cannot distinguish between dimers and higher order complexes of oligomers, such as tetramers. The strategy was to inhibit dimerization by modifying putative dimer contacts and to compare the functional properties of modified variants with those of WT MC4R. The strategy of dimer dissociation has also been used several times for other GPCRs and can shift the monomer-dimer equilibrium toward the monomeric state (Lambert 2010, Kasai et al. 2011).

\section{Materials and methods}

\section{Construction of chimeric MC4R/CB1R receptors and the MC4R variants His158Arg and His158Ala}

Mutant receptors were constructed by site-directed mutagenesis and chimeric receptors using PCR with overlapping oligonucleotides, with MC4R and cannabinoid-1 receptor (CB1R) as full-length templates in the pcDps expression vector (kindly provided by Prof. Torsten Schöneberg (University of Leipzig)). Full-length chimeric receptors were then either N-terminally hemagglutinin (HA)-tagged (NHA) or C-terminally FLAG-tagged (FLAG) or received both tags (HAF). Additionally, a naturally occurring MC4R variant His158Arg (Hinney et al. 2006) (located in the intracellular loop 2 (ICL2)) was investigated with respect to dimerization. For comparison, we also designed a histidine-to-alanine substitution at this position (His158Ala) by site-directed mutagenesis.

\section{Cell culture and transfection}

All functional studies were performed in COS-7 cells. COS-7 cells were maintained in DMEM (Biochrom, Berlin, Germany), supplemented with 10\% FCS (PAA Laboratories $\mathrm{GmbH}$, Cölbe, Germany), 2 mM L-glutamine (Invitrogen), $100 \mathrm{units} / \mathrm{ml}$ penicillin, and $100 \mu \mathrm{g} / \mathrm{ml}$ streptomycin (Biochrom). Cells were incubated at $37^{\circ} \mathrm{C}$ in humidified air containing $5 \% \mathrm{CO}_{2}$. Transfections were carried out $24 \mathrm{~h}$ after seeding using metafectene (Biontex, Munich, Germany), according to the manufacturer's protocol. Total receptor expression and receptor interaction were determined by a sandwich ELISA with $5 \times 10^{5}$ cells seeded in $6 \mathrm{~cm}$ dishes and transfected with a total of $3 \mu \mathrm{g}$ DNA and $8 \mu \mathrm{l}$ metafectene. Cell surface expression assays and cAMP assays were performed in 48 -well plates. Cell surface expression was determined with $5 \times 10^{4}$ cells/well transfected with $0.17 \mu \mathrm{g}$ DNA/well and $1 \mu \mathrm{l}$ metafectene/well. Intracellular cAMP accumulation was performed with $4 \times 10^{4}$ cells/well, and transfected with $0.08 \mu \mathrm{g}$ DNA/well and $0.93 \mu \mathrm{l}$ metafectene/well. Signaling studies were performed with N-terminally HA-tagged constructs. Further tests indicated that the signaling properties are not affected by the addition of tags.

\section{Determination of chimeric and mutant receptor cell surface expression}

Seventy-two hours after transfection with N-terminally HA-tagged receptors, cells were washed with Dulbecco's PBS (DPBS, Biochrom) and fixed for $30 \mathrm{~min}$ in $4 \%$ formaldehyde in DPBS, followed by washing twice in DPBS. After incubation in blocking buffer (10\% FCSsupplemented DMEM) for $1 \mathrm{~h}$ at $37^{\circ} \mathrm{C}$, cells were incubated for $2 \mathrm{~h}$ at $37^{\circ} \mathrm{C}$ in blocking buffer with $1 \mu \mathrm{g} / \mathrm{ml}$ biotinlabeled anti-HA MAB (Roche). After four washing steps in DPBS, the samples were incubated at $37^{\circ} \mathrm{C}$ overnight in blocking buffer with $1 \mu \mathrm{g} / \mathrm{ml}$ streptavidin-labeled

Published by Bioscientifica Ltd. 
peroxidase (BioLegend, San Diego, CA, USA). After four washing steps, the samples were stained with $0.1 \% \mathrm{H}_{2} \mathrm{O}_{2}$ and $10 \mu \mathrm{g} o$-phenylenediamine (Sigma-Aldrich) in substrate buffer (0.1 M citric acid, and $0.1 \mathrm{M} \mathrm{Na}_{2} \mathrm{HPO}_{4}$ at $\mathrm{pH}$ 5.2). The reaction was stopped after $10 \mathrm{~min}$ with $1 \mathrm{M} \mathrm{Na}_{2} \mathrm{SO}_{3}$ in $1 \mathrm{M} \mathrm{HCl}$. Colorimetry was carried out using an Anthos reader 2001 (Anthos Labtec Instruments GmbH, Salzburg, Austria), employing absorption at $492 \mathrm{~nm} / 620 \mathrm{~nm}$.

\section{Determination of total receptor expression and receptor-receptor interaction by ELISA}

To detect total expression, cells were transfected with N-terminally HA- and C-terminally FLAG-tagged (HAF) constructs. To detect dimerization capacity, cells were co-transfected with equal amounts of N-terminally HA-tagged constructs and C-terminally FLAG-tagged constructs. Seventy-two hours after transfection, cells were washed twice with cold DPBS, harvested, and solubilized overnight at $4{ }^{\circ} \mathrm{C}$ in lysis buffer $(10 \mathrm{mM}$ Tris/ $\mathrm{HCl}, 150 \mathrm{mM}$ $\mathrm{NaCl}, 1 \mathrm{mM}$ EDTA, $1 \mathrm{mM}$ dithiothreitol, $1 \%$ desoxycholate $\mathrm{Na}, 1 \% \mathrm{NP}-40$, and $0.2 \mathrm{mM}$ phenylmethylsulphonyl fluoride in water). The lysates were incubated in anti-FLAG antibody (Sigma-Aldrich)-coated 96-well plates for $2 \mathrm{~h}$. Following five washing steps, the samples were incubated at $37^{\circ} \mathrm{C}$ for $2 \mathrm{~h}$ in DPBS-T (DPBS with $0.05 \%$ Tween 20 ) with $1 \mu \mathrm{g} / \mathrm{ml}$ biotin-labeled anti-HA MAB (Roche). This step was followed by four washing steps with DPBS-T and incubation at $37^{\circ} \mathrm{C}$ for $1 \mathrm{~h}$ in blocking buffer with $1 \mu \mathrm{g} / \mathrm{ml}$ streptavidinlabeled peroxidase (BioLegend). The color reaction was performed as described earlier. The protein concentration of cell lysates of each sample was measured with the BCA Protein Assay Reagent Kit (Thermo Scientific, Bonn, Germany), according to the manufacturer's protocol. Results for total receptor expression and receptor-receptor interaction were calculated in relation to protein content. Due to the determination of dimer formation with differentially tagged receptors, the following GPCR-tag interaction combinations are possible: GPCR-NHA+ GPCR-NHA, GPCR-FLAG + GPCR-FLAG, and dimers with both GPCR-tags GPCR-NHA+GPCR-FLAG. The ELISA only detects one specific combination of the three: the GPCR-NHA+GPCR-FLAG dimers. Therefore, only one third of dimers were detectable in principle according to this method.

\section{Measurement of CAMP accumulation}

Forty-eight hours after transfection, cells were pre-incubated for 5 min with stimulation buffer (serum-free DMEM and $1 \mathrm{mM}$ 3-isobutyl-1-methylxanthine (IBMX, SigmaAldrich). This stimulation buffer was used for all further steps. Cells were then stimulated for $45 \mathrm{~min}$ at $37^{\circ} \mathrm{C}$ with $100 \mathrm{nM}$ (Nle4, D-Phe7)- $\alpha$-MSH (NDP- $\alpha-\mathrm{MSH}$, SigmaAldrich) or $1000 \mathrm{nM} \alpha$-MSH (Sigma-Aldrich, in the case of His158Arg and His158Ala) in stimulation buffer. Ligands were dissolved in DPBS with $0.1 \%$ BSA. The reaction was stopped by aspiration of medium. Cells were lysed at $4{ }^{\circ} \mathrm{C}$ for $1 \mathrm{~h}$ on a shaking platform with cell lysis buffer containing $5 \mathrm{nM}$ HEPES, $0.1 \%$ BSA, $0.3 \%$ Tween 20 , and $1 \mathrm{mM}$ IBMX. Each sample was transferred to a 384-well plate (Perkin-Elmer Life Science, Inc., Boston, MA, USA) and intracellular cAMP was determined using the AlphaScreen technology manufacturer's protocol (Perkin-Elmer; Staubert et al. 2007). The plate was measured with the Berthold Microplate Reader (Berthold Technologies GmbH \& Co. KG, Bad Wildbad, Germany).

\section{Structural MC4R homology model}

A structural homology model of the monomeric MC4R was generated as described previously (Tarnow et al. 2008). Sequence alignment was used to define helical and loop regions - as presented in the supporting information (supporting information, Supplementary Figure S1, see section on supplementary data given at the end of this article), in combination with helix assignments in GPCR crystal structures (Worth et al. 2009). Structure images were produced using PyMOL software (DeLano WL, version 1.03, San Carlos, CA, USA).

\section{Statistical analysis}

Data and statistical analyses were performed using the statistical tools (unpaired two-tailed $t$-test, if necessary with Welch's correction) implemented in Graph Pad Prism Version 5 (GraphPad Software, San Diego, CA, USA).

\section{Results}

The general strategy used here was to inhibit MC4R dimerization by substituting different amino acids into the potential dimeric interface. This exploited the fact that the MC4R and the CB1R - which was used for constructing chimeric receptors - are not able to form dimers (Rediger et al. 2009). Chimeric MC4R/CB1R variants with specific multiple substitutions between transmembrane helix 3 (TMH3)-TMH4, including ICL2, were designed as described in Fig. 1. The structural dimensions of variations were derived from the sequence and by comparing the

Published by Bioscientifica Ltd. 


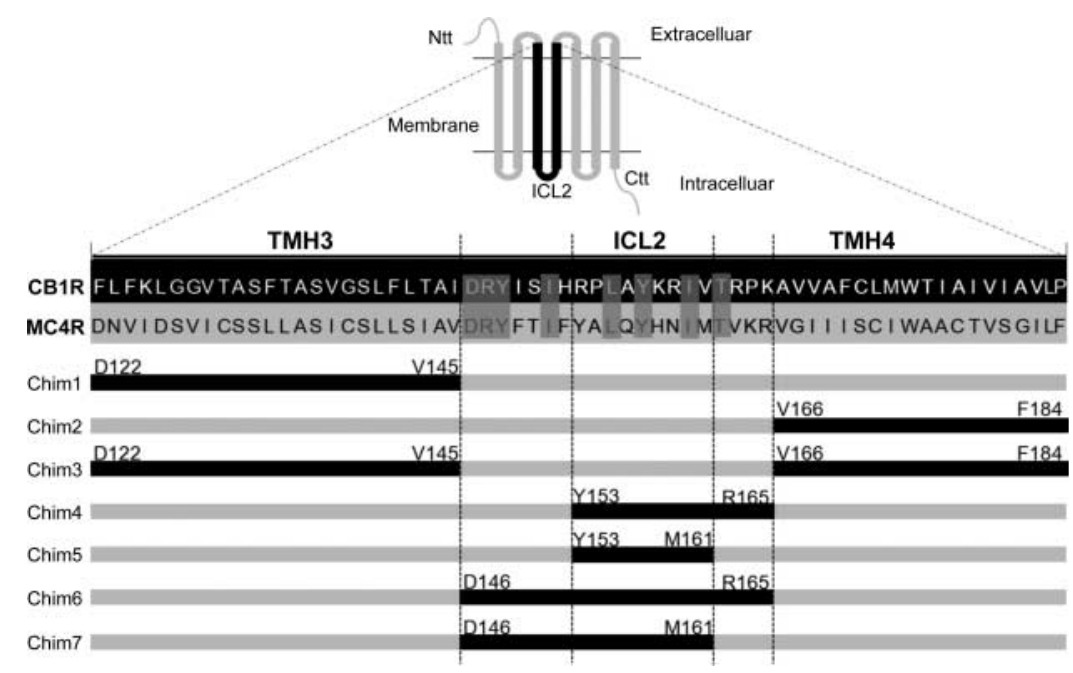

\section{Figure 1}

Assignment of chimeric MC4R/CB1R receptor constructs. MC4R/CB1R chimeric receptors were constructed via overlap PCR. The details of the MC4R (gray) regions substituted by the section of the CB1R (black) are described and shown in detail. The assigned amino acid regions are based on MC4R sequence alignment (supporting information, Supplementary Figure S1) and structural insights from GPCR crystal structures. Identical amino acids are shaded gray at the TMH3-ICL2-TMH4 junction, in order to highlight the 12 amino acids within this region that differ between the receptors. Chimera specification: chim1, substitution of TMH3

structures of different GPCRs (supporting information, Supplementary Figure S1). The reason this experimental setup was chosen is that several GPCR-GPCR interfaces have been reported to be located in this region (Carrillo et al. 2004, Guo et al. 2008, Mancia et al. 2008). Furthermore, it has been shown that CB1R not only signals via Gi but also via Gs activation (Rhee et al. 1998), indicating that the substitutions performed most probably do not modify MC4R G-protein specificity. The signaling properties of our chimeric MC4R/CB1R constructs and two single side chain substitutions were tested in comparison with the WT MC4R (for an overview, all results are summarized in the supporting information, Supplementary Table S1, see section on supplementary data given at the end of this article).

\section{Substitution of the entire TMH3 or TMH4 impair receptor cell surface expression levels}

Despite the fact that chimeras 1, 2, and 3 (chim1, chim2, and chim3) with fully substituted TMH3 and/or TMH4 showed total expression levels comparable to WT MC4R, the cell surface expression of these constructs was greatly decreased (Fig. 2A and B). Functional characterization also showed that the signaling capacity was totally abrogated
(Asp122-Val145); chim2, substitution of TMH4 (Val166-Phe184); chim3, substitution of TMH3 (Asp122-Val145) and TMH4 regions (Val166-Phe184); chim4, substitution of the ICL2 and intracellularly located regions of TMH4 (Tyr153-Arg165); chim5, substitution of ICL2 (Tyr153-Met161); chim6, substitution of the intracellularly located region of TMH3, ICL2, and intracellularly located region of TMH4 (Asp146-Arg165); and chim7, substitution of the intracellularly located TMH3 and intracellular loop 2 (Asp146-Met161).

(supporting information, Supplementary Table S1). We therefore excluded these constructs from further experiments. Of note, it was reported in previous studies that specific amino acids in TMH3 are significant for MC4R function, including Asp122 and Asp125 (Pogozheva et al. 2005). Cell surface expression level of chim 4 was moderately reduced in comparison to chim5, chim6, chim7 and MC4R-WT (Fig. 2B).

\section{TMH3 and ICL2 constrain the inactive state}

MC4R/CB1R chimeras were tested for their signaling properties (Fig. 3). All constructs exhibited elevated basal cAMP levels compared with WT. Chim6 and chim7 are combinations of TMH3 and ICL2 substitutions (Fig. 1) and expressed the greatest constitutive activation of fourfold compared with WT.

The chimeric receptors with increased basal signaling activity also exhibited hyperstimulation after ligand treatment - by around 160-210\% compared with WT (Fig. 3). Moreover, chim4 exhibited decreased cell surface expression level and only slightly increased basal activity, as well as enhanced signaling capability comparable to WT. The typical concentration-response curve of chim7 revealed no shift in the $\mathrm{EC}_{50}$ value (supporting

Published by Bioscientifica Ltd 

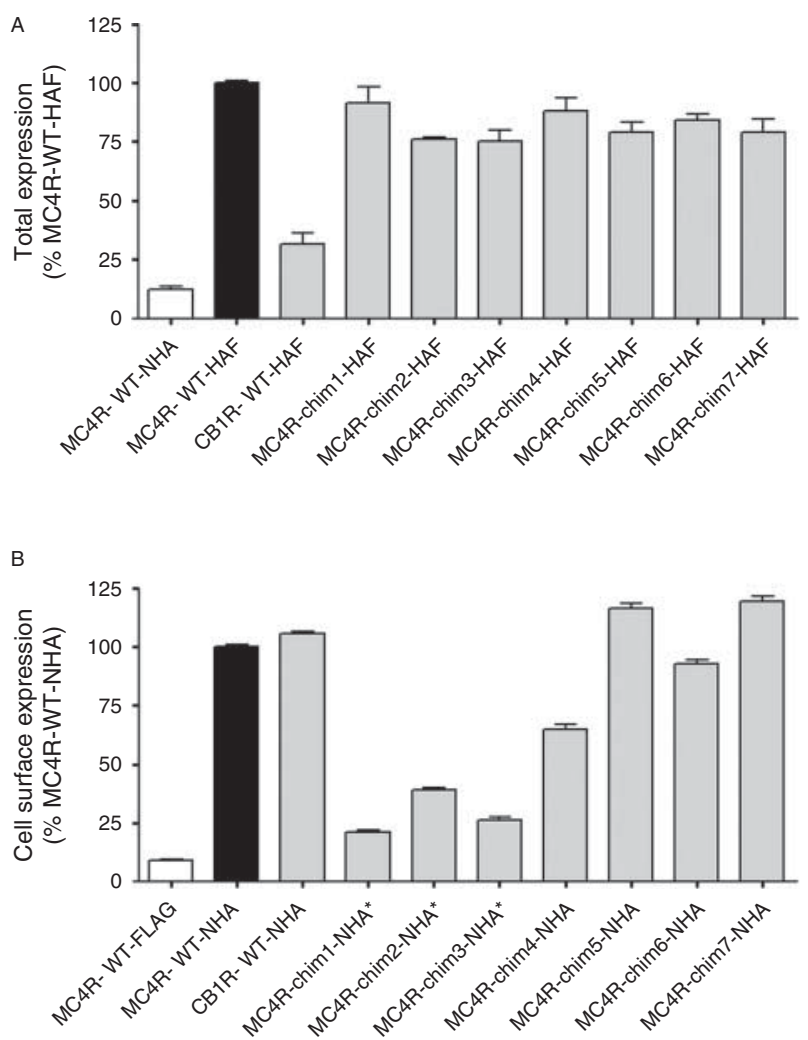

Figure 2

Expression of the chimeric MC4R/CB1R receptors. (A) Total receptor expression was determined in COS-7 cells with $\mathrm{N}$-terminally HA- and C-terminally FLAG-tagged (HAF) constructs using a sandwich ELISA approach. $\mathrm{N}$-terminally HA-tagged MC4R-WT served as negative control (MC4R-WT-NHA, white bar). The CB1R shows decreased total expression levels compared with the MC4R-WT (32\%). The MC4R/CB1R chimeras have comparable total expression levels. The values are calculated per $\mathrm{mg} / \mathrm{ml}$ protein and shown as percentage of the MC4R-WT expression (absorption (492/620) per $\mathrm{mg} / \mathrm{ml}$ protein: $0.9 \pm 0.21$ ). Data are means \pm s.E.M. of three or more independent experiments performed in triplicates. (B) For determination of cell surface expression, $\mathrm{N}$-terminally HA-tagged receptors were measured in intact COS-7 cells. The MC4R-WT C-terminally FLAGtagged served as negative control (MC4R-WT-FLAG, white bar). The chimeras chim1, chim2, and chim3 showed impaired and chim4 slightly decreased cell surface expression. We therefore excluded chim1, chim2, and chim3 from further functional experiments (marked with *). Values are given as percent of MC4R-WT expression (absorption (492/620): $0.6 \pm 0.18$ set as $100 \%)$. Data are means \pm s.E.M. of three or more independent experiments performed in six replicates.

information, Supplementary Figure S2, see section on supplementary data given at the end of this article).

The enhanced signaling of chim5-chim7 might also be due to signaling from MC4R homodimer to PTXsensitive G-proteins (Buch et al. 2009); however, signaling from MC4R to other G-proteins than Gs depends on the cell system used (Breit et al. 2011). In COS-7 cells, no reduction in basal activity was observed for the MC4R mutant Asp90Asn after NDP- $\alpha$-MSH challenge (Biebermann et al. 2003), as has been found in HEK293 cells (Buch et al. 2009). We also tested PTX-sensitive signaling for MC4R-WT in COS-7 cells and found no enhancement of cAMP accumulation (supporting information, Supplementary Figure S3, see section on supplementary data given at the end of this article). Therefore, in the cell system used, we have been able to exclude the possibility that cAMP hyperstimulation of chim5-chim7 is due to loss of PTX-sensitive G-protein activation.

\section{Specific regions of TMH3, TMH4, and ICL2 are important for MC4R homodimer formation}

To investigate the interaction between chimeric receptors, we performed a sandwich ELISA. All tested constructs included ICL2 substitution from CB1R into the MC4R and gave rise to decreased or impaired dimerization capacity (dimer dissociation). Chim 4 and chim6 showed significant decreases (69 and 67\% dimerization of WT, $P<0.0001)$ and chim5 and chim7 highly significant reductions (39 and $31 \%$ dimerization of WT, $P<0.0001$ ) in homodimerization capacities compared with WT (Fig. 4, in addition homodimerization capacities chim1chim3 supporting information, Supplementary Figure S4, see section on supplementary data given at the end of this article). It is striking that chim 4 and chim6, with the lowest suppression of dimer formation, included substitution of both CB1R ICL2 and the junction of TMH4. Chim5 and chim7 exhibited the maximum suppression of

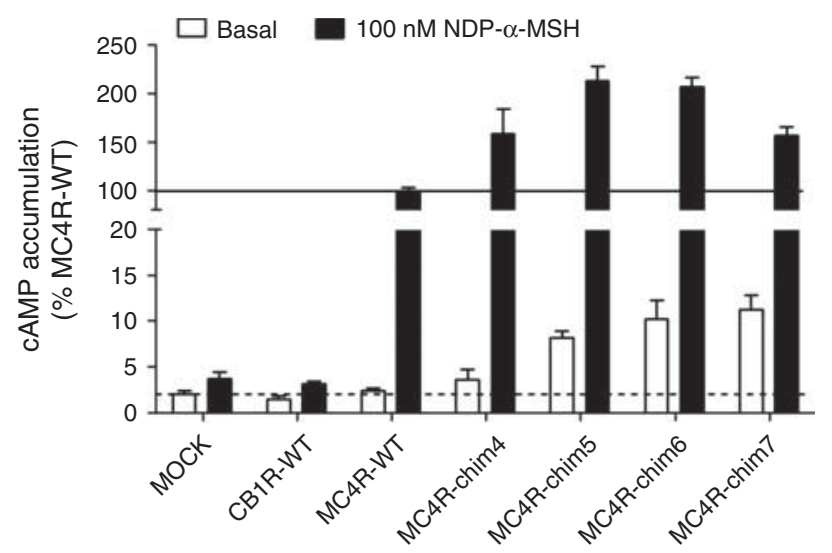

Figure 3

Signaling of the chimeric MC4R/CB1R receptors. CAMP accumulation was measured in COS-7 cells using the AlphaScreen technology. Cells were stimulated with $100 \mathrm{nM}$ NDP- $\alpha$-MSH. All tested chimeras show increased basal and ligand-induced CAMP accumulation compared with the MC4R-WT, with the exception of chim4, which had no significant increase in ligand-independent activity. The empty pcDps-vector served as negative control (MOCK) marked with dashed line. Values are given in percentage of MC4R-WT signaling marked with solid line (MC4R-WT 93.7 $\pm 11.94 \mathrm{nM}$ was set as $100 \%$ ). Data are means \pm s.E.M. of three independent experiments performed in triplicates. 


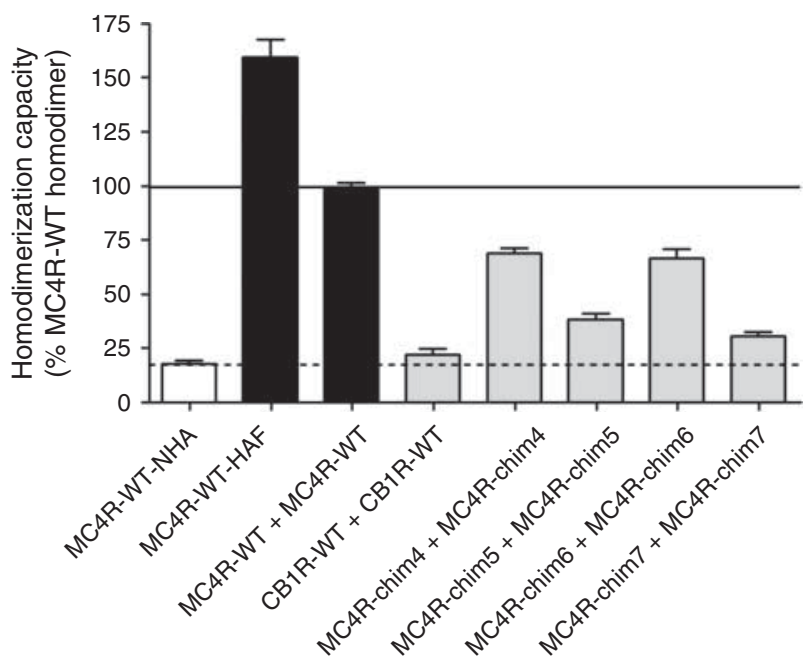

Figure 4

Dimerization of the chimeric MC4R/CB1R receptors. To investigate dimerization by sandwich ELISA, COS-7 cells were transiently co-transfected with equal amounts of $\mathrm{N}$-terminally $\mathrm{HA}$-tagged constructs and C-terminally FLAG-tagged constructs. The FLAG-tagged receptors attach to the FLAGantibody-coated 96-well plate. The dimerization capacities were measured via the $\mathrm{N}$-terminally $\mathrm{HA}$-tagged receptor as an increase in optical density (mean absorption (492/620)). The N-terminally HA-tagged MC4R transfected alone served as negative control (MC4R-WT-NHA, white bar). $\mathrm{N}$-terminally HA- and C-terminally FLAG-tagged MC4R (MC4R-WT-HAF) and the MC4R-WT homodimer (MC4R-WT-NHA + MC4R-WT-FLAG) served as positive controls (black bars). The CB1R is not able to form homodimers (Rediger et al. 2009). Chim4 and chim6 show slightly decreased (69 and $67 \%$ ) and chim5 and chim7 greatly decreased (39 and $31 \%$ ) dimerization capacity compared with WT. The values are calculated per $\mathrm{mg} / \mathrm{ml}$ protein and shown as percentage of the MC4R-WT homodimer (absorption $(492 / 620) / \mathrm{mg} / \mathrm{ml}$ protein: $0.6 \pm 0.14)$. Data are means \pm s.E.M. of four or more independent experiments performed in triplicates.

dimer formation and were modified either in ICL2 alone or in ICL2 and the proximate region to TMH3.

To ensure that the sandwich ELISA data are reproducible with a second method, we applied a YFP-based bimolecular fluorescence complementation assay (BiFC), which permits the determination of receptor interaction in living cells (supporting information, Supplementary Figure S5 and S6). As an example, we tested interactions for chim 6 and chim7 and demonstrated once again that homodimerization of chim 6 and chim 7 is reduced compared with dimerization of MC4R-WT.

\section{The naturally occurring MC4R mutation His158Arg in ICL2 exhibits enhanced signaling properties and reduced dimer formation}

We also characterized the naturally occurring MC4R variant His158Arg in ICL2 (Hinney et al. 2006) with expression parameters comparable to WT (supporting information, Supplementary Table S1). This mutation revealed a threefold increased basal level of cAMP accumulation (Fig. 5A). Maximal ligand-induced signaling was increased to $168 \%$ of WT. To confirm the importance of this position and to investigate how these findings depend on side chain properties, we also designed and tested the His158Ala substitution. We detected a threefold increase in basal levels of cAMP accumulation and enhanced ligand-induced signaling.

In addition, the naturally occurring constitutively activating mutation (CAM) His158Arg and the variant His158Ala exhibited decreased homodimerization, corresponding to $56-64 \%$ of the value for the WT (Fig. $5 B$ ). On the one hand, this clearly indicates that inhibition of dimerization by modification at this position does not depend on the substituted side chain. On the other hand, it appears that the ICL2 is indeed a crucial key region for dimeric organization.

\section{Discussion}

In this study, we aimed to investigate two principle aspects of MC4R: i) the structural regions participating in dimerization and ii) the functional properties of receptor constructs with depressed dimerization capacity. We have found that both multiple and single substitutions in the intracellular region of the receptor of TMH3-ICL2-TMH4 inhibit MC4R dimerization (Fig. 6). From these results, we conclude that ICL2 makes the major contribution to the MC4R homodimerization process, with respect to our experimental setup. In accordance with this, our group has recently shown that disulphide bridges are not involved in MC4R dimerization (Elsner et al. 2006), which has been suggested for other GPCRs (Berthouze et al. 2007). However, we cannot exclude the possibility that TMH1 and TMH2, or TMH5-7, might play an additional role in MC4R-MC4R interactions, as was recently speculated for MC4R and as has been suggested for other GPCRs in general (Lee et al. 2003).

It is known that GPCRs probably exist in a monomerdimer equilibrium ((Kasai et al. 2011), reviewed in Lambert (2010)), which raises the possibility that specific modifications in the receptors might shift the equilibrium toward one of these states. There have only been a few reports (Grant et al. 2004, Ravindran et al. 2009, Moreno et al. 2012) of GPCR dimer dissociation induced by ligand binding or mutations. Single side chain substitutions have been found to interrupt the dimer interface at TMH4, as in chemokine receptor CCR5 (one residue) or the heterodimer between the metabotropic glutamate- 2 receptor and

Published by Bioscientifica Ltd 

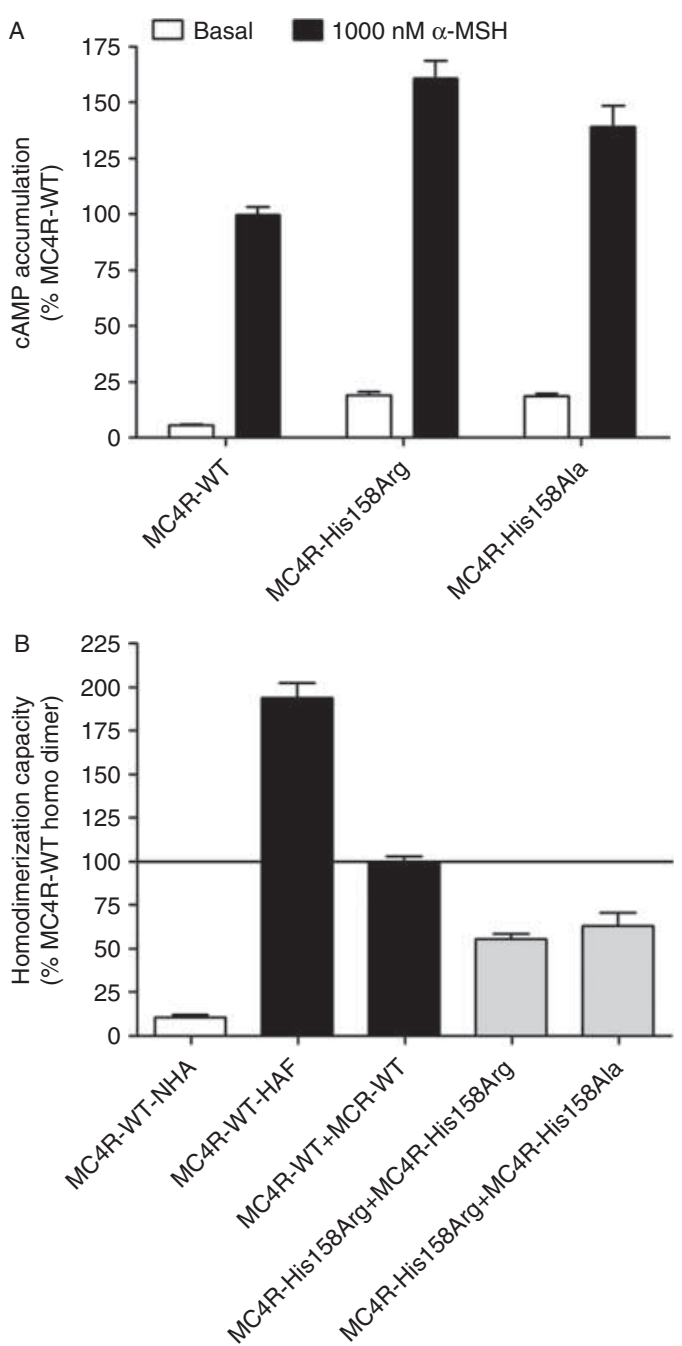

Figure 5

Functional investigation of the pathogenic MC4R mutation His158Arg and the artificial mutation His158Ala. (A) CAMP accumulation was measured in COS-7 cells using the AlphaScreen technology. Cells were stimulated with $1000 \mathrm{nM}$ of the endogenous ligand $\alpha-\mathrm{MSH}$. Both mutants shows increased basal and ligand-induced CAMP accumulation compared with the MC4RWT. Values are given in percentage of MC4R-WT signaling (MC4R-WT $153.5 \pm 34.10 \mathrm{nM}$ was set as $100 \%$ ). Data are means \pm s.E.M. of three independent experiments performed in triplicates. (B) Dimerization of MC4R mutant His158Arg and His158Ala was investigated using transiently co-transfected COS-7 cells with equal amounts of N-terminally HA-tagged constructs and C-terminally FLAG-tagged constructs. The FLAG-tagged receptor attached to the FLAG antibody-coated 96-well plate. The dimerization capacities were measured via the $\mathrm{N}$-terminally $\mathrm{HA}$-tagged receptor as an increase in optical density (mean absorption (492/620)). The $\mathrm{N}$-terminally HA-tagged MC4R served as negative control (MC4R-WT-NHA, white bar). N-terminally HA- and C-terminally FLAG-tagged MC4R (MC4RWT-HAF) and the MC4R-WT homodimer (MC4R-WT-NHA + MC4R-WT-FLAG) served as positive controls (black bars). Both MC4R-His158Arg and MC4RHis158Ala show decreased (56 and 64\%) dimerization capacity compared with MC4R-WT. The values are calculated per $\mathrm{mg} / \mathrm{ml}$ protein and shown as percentage of the MC4R-WT homodimer (absorption (492/620)/mg/ml protein: $0.3 \pm 0.03$ ). Data are means \pm S.E.M. of four independent experiments performed in triplicates. the serotonin-5 receptor (three residues) (Hernanz-Falcon et al. 2004, Moreno et al. 2012).

We also detected an increase in the permanent (basal) signaling activity for single and multiple substitutions in ICL2 and adjacent helices. It is well known for MC4R that particular mutations are able to increase the constitutive (ligand independent) basal tone of signaling (cAMP accumulation), such as mutations at Leu140 in TMH3 (Mo et al. 2012). It has also been reported that specific mutations may induce biased signals, whereby cAMP accumulation related to Gs and to the pERK1/2 pathway can be differentially modified (Mo et al. 2012). Moreover, a systematic and comprehensive mutagenesis study on amino acids of TMH6 revealed further significant insights into signaling regulation at MC4R (Huang \& Tao 2012). Several specific mutations in TMH6 induced constitutive activation of pERK1/2 phosphorylation, or constitutive activation of Gs, as well as inactivating mutations were identified. Taken together, these findings provide detailed information on the determinants of the MC4R mechanism in the transmembrane region (Huang \& Tao 2012). Finally, such systematic approaches help to elucidate intramolecular interaction networks and help us to understand in detail how the receptor switches between the different activity states. In addition, the $\mathrm{N}$-terminus of MC4R is also important in the regulation of ligand-independent signaling. The results of mutagenesis at specific residues in the N-terminal MC4R region had suggested that this region acts as an intramolecular, tethered agonist (partial) and maintains a basal signaling tone (Srinivasan et al. 2004). Specific naturally occurring mutations at the N-terminal tail decreased the level of basal cAMP accumulation, which also highlights the importance of this signaling capacity under pathophysiological conditions.

Our current study is compatible with the concept that the degree of oligomerization could also be involved in the regulation of the basal signaling activity. This is indicated by the simultaneous occurrence of dimer separation and increased basal activity. For further studies, it might be of interest to investigate the function of the N-terminus in combination with CAMs at other parts of the receptor. In summary, the important basal activity level of MC4R can be regulated at different receptor regions and tuned up or down, probably by a variety of mechanisms.

It is striking that ligand-induced Gs-mediated signaling capacity was enhanced in our constructs with dissociated dimers. This may be caused by synergy between the increased basal activity and the capacity of ligand-induced stimulation. Alternatively, this finding

Published by Bioscientifica Ltd 


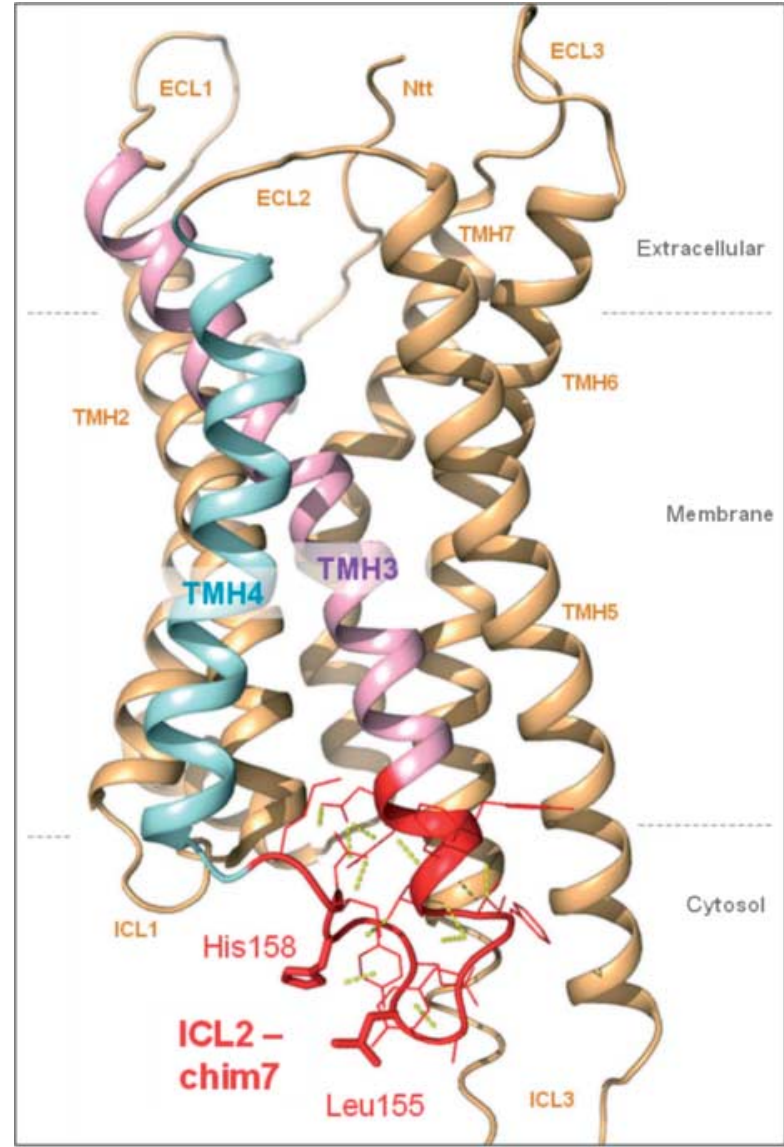

\section{Figure 6}

Structural implications from a MC4R homology model. Our aim was to induce MC4R dimer dissociation by substituting specific $C B 1 R$ fragments into the potential MC4R-MC4R dimer interface, in order to identify protomer-protomer contacts and to compare functional properties between monomeric vs dimeric MC4R. Therefore, it was important to maintain the capacity of chimeric receptors to couple Gs such as wild-type MC4R and to avoid modifying the coupling specificity of MC4R for Gs by constructing chimeric receptors. The CB1R is a Gi- and Gs protein coupling GPCR (Howlett et al. 1986) and is known from GPCRs such as the $\beta$-adrenergic receptor 2 (ADRB2) that the essential amino acids for $\mathrm{Gi}$ and Gs coupling are similar. One of these residues is Phe139 at the ICL2 of ADRB2, which is leucine 155 at the corresponding position of MC4R. Both amino acids are hydrophobic and conservation of Phe/Leu residues at this position has been reported, based on comparison of different family A GPCRs (Rasmussen et al. 2011). In the chimeric receptor variants MC4R/CB1R, a leucine is also localized at the corresponding position (supporting information, Supplementary Figure S1). This indicates that the Gs coupling capacity at this position is also probably maintained in the chimeric receptors. Substituted helices or receptor regions (e.g. MC4R chim7) are highlighted by different colors in the structural MC4R homology model. Our experimental data suggest that the MC4R homodimeric constellation is partially inhibited by substitutions at the transition between ICL2 and TMH3 (red). In the natural MC4R variant His158Arg tested here, it is of special note that the corresponding CB1R amino acid is Lys225 (CB1R sequence number), which is also positively charged like the arginine MC4R variant. The solid line marked homodimer formation of the MC4R-WT. This single substitution, as well as the variant His158Ala, leads to partial dissociation of MC4R dimers, thus confirming the importance of MC4R ICL2 for dimeric organization. might also be explained by the receptor:G-protein ratio of 2:1 in the homodimeric WT, in contrast to the ratio of 1:1 between increased levels of MC4R monomers and G-proteins. However, our data and experimental setup do not provide clear evidence that there is a direct relationship between homodimerization/monomerization and the capacity for signaling. This question can be studied in future with heterodimeric MC4R/GPCR complexes. Several studies on different GPCRs have found significant differences between GPCR-GPCR dimers and monomers with respect to structure-function relationships: i) for homodimers of dopamine receptors, it has been shown (Guo et al. 2005) that TMH4 orientation is modified during (dimeric) receptor activation; ii) studies on homodimeric GPCRs have found that, after agonist-mediated activation, G-protein coupling may occur at only one protomer (Damian et al. 2006); and iii) conformational changes between $\alpha_{2} \mathrm{~A}$-adrenergic and $\mu$-opioid receptor heterodimers have been reported, whereby the propagated conformational change from one activated receptor caused inactivation of the second receptor (Vilardaga et al. 2008).

In summary, these findings support a concept whereby the ICL2, together with specific adjacent regions of TMH3 and TMH4, is important for constituting a dimeric MC4R constellation. Modifications here lead to dissociation of the dimers into receptor monomers. Furthermore, this region is also important for maintaining an inactive state and for determining signaling capacity. In conclusion, the conformation and interplay of the entire intracellular TMH3-ICL2-TMH4 region is a regulating element in signaling and receptor organization. Our findings add to the few known examples of GPCR dimer dissociation induced by ligand binding or mutations (Grant et al. 2004, Ravindran et al. 2009, Moreno et al. 2012).

\section{Supplementary data}

This is linked to the online version of the paper at http://dx.doi.org/10.1530/ JME-13-0061.

\section{Declaration of interest}

The authors declare that there is no conflict of interest that could be perceived as prejudicing the impartiality of the research reported.

\section{Funding}

This work was funded by the Deutsche Forschungsgemeinschaft (BI 893/2-1, BI893/5-1, BI893/6-2) and graduate school 1208: Hormonal Regulation of Energy Metabolism, Body Weight and Growth, TP1.

Published by Bioscientifica Ltd 


\section{Author contribution statement}

$C L P, H B, G K$, and $A R$ were involved in conception and design of the experiments. $C L P, A R, C L, J M, P T$, and $A M$ were involved in performance of the experiments. CLP, H B, G K, JP, A M, A G, and $H K$ were involved in data analysis. All authors were involved in writing the paper and were responsible for the final approval of the submitted and published versions.

\section{Acknowledgement}

The authors are grateful for the important contributions of Lara Zajic.

\section{References}

Berthouze M, Rivail L, Lucas A, Ayoub MA, Russo O, Sicsic S, Fischmeister R, Berque-Bestel I, Jockers R \& Lezoualc'h F 2007 Two transmembrane Cys residues are involved in 5-HT4 receptor dimerization. Biochemical and Biophysical Research Communications 356 642-647. (doi:10.1016/j.bbrc. 2007.03.030)

Biebermann H, Krude H, Elsner A, Chubanov V, Gudermann T \& Gruters A 2003 Autosomal-dominant mode of inheritance of a melanocortin-4 receptor mutation in a patient with severe early-onset obesity is due to a dominant-negative effect caused by receptor dimerization. Diabetes $\mathbf{5 2}$ 2984-2988. (doi:10.2337/diabetes.52.12.2984)

Biebermann H, Kuhnen P, Kleinau G \& Krude H 2012 The neuroendocrine circuitry controlled by POMC, MSH, and AGRP. Handbook of Experimental Pharmacology 209 47-75. (doi:10.1007/978-3-642-24716-3_3)

Breit A, Buch TR, Boekhoff I, Solinski HJ, Damm E \& Gudermann T 2011 Alternative $G$ protein coupling and biased agonism: new insights into melanocortin-4 receptor signalling. Molecular and Cellular Endocrinology 331 232-240. (doi:10.1016/j.mce.2010.07.007)

Buch TR, Heling D, Damm E, Gudermann T \& Breit A 2009 Pertussis toxinsensitive signaling of melanocortin-4 receptors in hypothalamic GT1-7 cells defines agouti-related protein as a biased agonist. Journal of Biological Chemistry 284 26411-26420. (doi:10.1074/jbc.M109.039339)

Carrillo JJ, Lopez-Gimenez JF \& Milligan G 2004 Multiple interactions between transmembrane helices generate the oligomeric $\alpha 1 \mathrm{~b}$-adrenoceptor. Molecular Pharmacology 66 1123-1137. (doi:10.1124/mol.104. 001586)

Cone RD 2006 Studies on the physiological functions of the melanocortin system. Endocrine Reviews 27 736-749. (doi:10.1210/er.2006-0034)

Damian M, Martin A, Mesnier D, Pin JP \& Baneres JL 2006 Asymmetric conformational changes in a GPCR dimer controlled by G-proteins. EMBO Journal 25 5693-5702. (doi:10.1038/sj.emboj.7601449)

Elsner A, Tarnow P, Schaefer M, Ambrugger P, Krude H, Gruters A \& Biebermann H 2006 MC4R oligomerizes independently of extracellular cysteine residues. Peptides 27 372-379. (doi:10.1016/j.peptides.2005. 02.027)

Farooqi IS, Yeo GS \& O'Rahilly S 2003 Binge eating as a phenotype of melanocortin 4 receptor gene mutations. New England Journal of Medicine 349 606-609 author reply 606-609. (doi:10.1056/ NEJM200308073490615)

George SR, O'Dowd BF \& Lee SP 2002 G-protein-coupled receptor oligomerization and its potential for drug discovery. Nature Reviews. Drug Discovery 1 808-820. (doi:10.1038/nrd913)

Grant M, Collier B \& Kumar U 2004 Agonist-dependent dissociation of human somatostatin receptor 2 dimers: a role in receptor trafficking. Journal of Biological Chemistry 279 36179-36183. (doi:10.1074/jbc. M407310200)

Guo W, Shi L, Filizola M, Weinstein H \& Javitch JA 2005 Crosstalk in $\mathrm{G}$ protein-coupled receptors: changes at the transmembrane homodimer interface determine activation. PNAS 102 17495-17500. (doi:10.1073/pnas.0508950102)
Guo W, Urizar E, Kralikova M, Mobarec JC, Shi L, Filizola M \& Javitch JA 2008 Dopamine D2 receptors form higher order oligomers at physiological expression levels. EMBO Journal 27 2293-2304. (doi:10.1038/emboj.2008.153)

Hernanz-Falcon P, Rodriguez-Frade JM, Serrano A, Juan D, del Sol A, Soriano SF, Roncal F, Gomez L, Valencia A, Martinez AC et al. 2004 Identification of amino acid residues crucial for chemokine receptor dimerization. Nature Immunology 5 216-223. (doi:10.1038/ni1027)

Hinney A, Bettecken T, Tarnow P, Brumm H, Reichwald K, Lichtner P, Scherag A, Nguyen TT, Schlumberger P, Rief W et al. 2006 Prevalence, spectrum, and functional characterization of melanocortin-4 receptor gene mutations in a representative population-based sample and obese adults from Germany. Journal of Clinical Endocrinology and Metabolism 91 1761-1769. (doi:10.1210/jc.2005-2056)

Howlett AC, Qualy JM \& Khachatrian LL 1986 Involvement of Gi in the inhibition of adenylate cyclase by cannabimimetic drugs. Molecular Pharmacology 29 307-313.

Huang H \& Tao YX 2012 Pleiotropic functions of the transmembrane domain 6 of human melanocortin-4 receptor. Journal of Molecular Endocrinology 49 237-248. (doi:10.1530/JME-12-0161)

Kasai RS, Suzuki KG, Prossnitz ER, Koyama-Honda I, Nakada C, Fujiwara TK \& Kusumi A 2011 Full characterization of GPCR monomer-dimer dynamic equilibrium by single molecule imaging. Journal of Cell Biology 192 463-480. (doi:10.1083/jcb.201009128)

Krishna R, Gumbiner B, Stevens C, Musser B, Mallick M, Suryawanshi S, Maganti L, Zhu H, Han TH, Scherer L et al. 2009 Potent and selective agonism of the melanocortin receptor 4 with MK-0493 does not induce weight loss in obese human subjects: energy intake predicts lack of weight loss efficacy. Clinical Pharmacology and Therapeutics 86 659-666. (doi:10.1038/clpt.2009.167)

Lambert NA 2010 GPCR dimers fall apart. Science Signaling 3 pe12. (doi:10.1126/scisignal.3115pe12)

Lee SP, O'Dowd BF, Rajaram RD, Nguyen T \& George SR 2003 D2 dopamine receptor homodimerization is mediated by multiple sites of interaction, including an intermolecular interaction involving transmembrane domain 4. Biochemistry 42 11023-11031. (doi:10.1021/bi0345539)

Levoye A, Dam J, Ayoub MA, Guillaume JL, Couturier C, Delagrange P \& Jockers R 2006 The orphan GPR50 receptor specifically inhibits MT1 melatonin receptor function through heterodimerization. EMBO Journal 25 3012-3023. (doi:10.1038/sj.emboj.7601193)

Mancia F, Assur Z, Herman AG, Siegel R \& Hendrickson WA 2008 Ligand sensitivity in dimeric associations of the serotonin 5HT2c receptor. EMBO Reports 9 363-369. (doi:10.1038/embor.2008.27)

Mo XL, Yang R \& Tao YX 2012 Functions of transmembrane domain 3 of human melanocortin-4 receptor. Journal of Molecular Endocrinology 49 221-235. (doi:10.1530/JME-12-0162)

Moreno JL, Muguruza C, Umali A, Mortillo S, Holloway T, Pilar-Cuellar F, Mocci G, Seto J, Callado LF, Neve RL et al. 2012 Identification of three residues essential for 5-HT2A-mGlu2 receptor heteromerization and its psychoactive behavioral function. Journal of Biological Chemistry $\mathbf{2 8 7}$ 44301-44319. (doi:10.1074/jbc.M112.413161)

Nickolls SA \& Maki RA 2006 Dimerization of the melanocortin 4 receptor: a study using bioluminescence resonance energy transfer. Peptides $\mathbf{2 7}$ 380-387. (doi:10.1016/j.peptides.2004.12.037)

Nijenhuis WA, Oosterom J \& Adan RA 2001 AgRP(83-132) acts as an inverse agonist on the human-melanocortin-4 receptor. Molecular Endocrinology 15 164-171. (doi:10.1210/me.15.1.164)

Pogozheva ID, Chai BX, Lomize AL, Fong TM, Weinberg DH, Nargund RP, Mulholland MW, Gantz I \& Mosberg HI 2005 Interactions of human melanocortin 4 receptor with nonpeptide and peptide agonists. Biochemistry 44 11329-11341. (doi:10.1021/bi0501840)

Rasmussen SG, DeVree BT, Zou Y, Kruse AC, Chung KY, Kobilka TS, Thian FS, Chae PS, Pardon E, Calinski D et al. 2011 Crystal structure of the $\beta 2$ adrenergic receptor-Gs protein complex. Nature $\mathbf{4 7 7} 549-555$. (doi:10.1038/nature10361) 
Ravindran A, Joseph PR \& Rajarathnam K 2009 Structural basis for differential binding of the interleukin-8 monomer and dimer to the CXCR1 N-domain: role of coupled interactions and dynamics. Biochemistry 48 8795-8805. (doi:10.1021/bi901194p)

Rediger A, Tarnow P, Bickenbach A, Schaefer M, Krude H, Gruters A \& Biebermann H 2009 Heterodimerization of hypothalamic G-protein-coupled receptors involved in weight regulation. Obesity Facts 2 80-86. (doi:10.1159/000209862)

Rediger A, Piechowski CL, Habegger K, Gruters A, Krude H, Tschop MH, Kleinau G \& Biebermann H 2012 MC4R dimerization in the paraventricular nucleus and GHSR/MC3R heterodimerization in the arcuate nucleus: is there relevance for body weight regulation. Neuroendocrinology 95 277-288. (doi:10.1159/000334903)

Rhee MH, Bayewitch M, Avidor-Reiss T, Levy R \& Vogel Z 1998 Cannabinoid receptor activation differentially regulates the various adenylyl cyclase isozymes. Journal of Neurochemistry 71 1525-1534. (doi:10.1046/j.1471-4159.1998.71041525.x)

Rozenfeld R \& Devi LA 2010 Receptor heteromerization and drug discovery. Trends in Pharmacological Sciences 31 124-130. (doi:10.1016/ j.tips.2009.11.008)

Rozenfeld R \& Devi LA 2011 Exploring a role for heteromerization in GPCR signalling specificity. Biochemical Journal 433 11-18. (doi:10.1042/ BJ20100458)

Srinivasan S, Lubrano-Berthelier C, Govaerts C, Picard F, Santiago P, Conklin BR \& Vaisse C 2004 Constitutive activity of the melanocortin-4 receptor is maintained by its $\mathrm{N}$-terminal domain and plays a role in energy homeostasis in humans. Journal of Clinical Investigation $\mathbf{1 1 4}$ 1158-1164. (doi:10.1172/JCI21927)

Staubert C, Tarnow P, Brumm H, Pitra C, Gudermann T, Gruters A, Schoneberg T, Biebermann H \& Rompler H 2007 Evolutionary aspects in evaluating mutations in the melanocortin 4 receptor. Endocrinology 148 4642-4648. (doi:10.1210/en.2007-0138)

Tao YX 2010 The melanocortin-4 receptor: physiology, pharmacology, and pathophysiology. Endocrine Reviews 31 506-543. (doi:10.1210/er. 2009-0037)

Tarnow P, Rediger A, Brumm H, Ambrugger P, Rettenbacher E, Widhalm K, Hinney A, Kleinau G, Schaefer M, Hebebrand J et al. 2008 A heterozygous mutation in the third transmembrane domain causes a dominant-negative effect on signalling capability of the MC4R. Obesity Facts 1 155-162. (doi:10.1159/000138251)

Van der Ploeg LH, Martin WJ, Howard AD, Nargund RP, Austin CP, Guan X, Drisko J, Cashen D, Sebhat I, Patchett AA et al. 2002 A role for the melanocortin 4 receptor in sexual function. PNAS 99 11381-11386. (doi:10.1073/pnas.172378699)

Vilardaga JP, Nikolaev VO, Lorenz K, Ferrandon S, Zhuang Z \& Lohse MJ 2008 Conformational cross-talk between $\alpha 2 \mathrm{~A}$-adrenergic and mu-opioid receptors controls cell signaling. Nature Chemical Biology 4 126-131. (doi:10.1038/nchembio.64)

Worth CL, Kleinau G \& Krause G 2009 Comparative sequence and structural analyses of G-protein-coupled receptor crystal structures and implications for molecular models. PLoS ONE 4 e7011. (doi:10.1371/ journal.pone.0007011)

Received in final form 10 May 2013

Accepted 14 May 2013

Accepted Preprint published online 14 May 2013
(C) 2013 Society for Endocrinology Printed in Great Britain 